\title{
Investigation of Behavior and Perception of Digital Library Users: A Cognitive Style Perspective
}

\author{
Enrique Frias-Martinez, Sherry Y. Chen ${ }^{*}$, Xiaohui Liu \\ School of Information Systems, Computing and Mathematics \\ Brunel University, Uxbridge, Middlesex, UB8 3PH, UK
}

\begin{abstract}
Cognitive style is an influential factor in users' information seeking. The study presented in this paper examines how users' cognitive styles affect their behavior and perception in digital libraries. Fifty participants took part in this study. Two dimensions of cognitive styles were considered: (a) Field Dependence/Independence; (2) Verbalizer/Imager. The results showed that Intermediate users and Verbalizers have not only more positive perception, but they also complete the tasks in effective ways. Implications for the design of personalized digital libraries are also discussed.
\end{abstract}

Keywords: Digital Libraries, Cognitive Styles, Individual Differences, User Modeling, Personalization

\section{Introduction}

Due to the diversity of information, Digital Libraries (DLs) have become one of the major web services (Liaw and Huang, 2003). Current DLs are becoming more complex systems than traditional libraries because DLs make information directly available to users via both Intranets and the Internet (Gonzalves and Fox, 2002). Using Intranets and the Internet to disseminate information, DLs allow serving broader audiences (Marchionini, Plaisant and Komlodi, 2003). In other words, users of DLs may have a greater variability, in terms of their background, knowledge and skills.

Nevertheless, most of current DLs take a global approach, by which all users are presented with the same interface. This global approach may make users feel difficult to locate information, because there is not a match between users' preferences and functionalities offered by DLs (Marchionini, Plaisant and Komlodi, 1998). This mismatch between user preferences and functionality design can be solved by providing personalization, which tailors content, structure, and/or presentation to match the unique and specific needs of each individual (Fink and Kobsa, 2000). In the past, personalization mainly focused on users' prior knowledge (Surjono, and Maltby, 2003). Recently, personalization has been made to try to accommodate the preferences of users with different cognitive styles (Mitchell, Chen, and Macredie, 2005). However, the delivery of personalization is complex because the adaptation to each individual requires the understanding of their preferences (Ardissono, et al., 2005) and prediction of their behavior (Deshpande and Karypis, 2004). Therefore, it is necessary to investigate how users with different cognitive styles interact with DLs so that effective personalization can be provided.

In this vein, the study presented in this paper investigates how cognitive styles affect the behavior and perception that user shows when interacting with DLs. The rest of the paper is organized as follows. In Section 2, we present previous related works which examined how cognitive styles affect users' information seeking. Section 3 describes the methodology used to conduct the study. Subsequently, the results related to user behavior and user perception are presented in Section 4. It then progresses to Section 5, which discusses the implications for system design based on the results obtained in Section 4. Finally, conclusions are drawn and possible directions for future works are identified in Section 6.

\footnotetext{
* Corresponding author: Tel: +44-1895266023; Fax: +44-1895-251686. Email address: Sherry.Chen@brunel.ac.uk
} 


\section{Theoretical Background}

Cognitive style refers to a user's information processing habits, representing an individual user's typical mode of perceiving, thinking, remembering, and problem solving (Messick 1976). Jonassen and Grabowski (1993) defined cognitive style as inbuilt and relatively consistent preferences in organizing and representing information. It is notable that there is a number of dimensions of cognitive styles, such as Holism/Serialism (Pask, 1976), Divergent/Convergent (Hudson, 1966), Field Dependence/Independence (Witkin et al., 1977), and Imager/Verbalizer (Riding, 1991). Among these, Field Dependence/Independence and Imager/Verbalizer are especially related to information seeking. The former concerns how users process and organize information whereas the latter emphasizes how users perceive the presentation of information.

Field Dependence/Independence is related to the degree to which a user's perception or comprehension of information is influenced by the context (Jonassen and Grabowski, 1993). The key issue of Field Dependence lies within the differences between Field Dependent and Field Independent users, which are presented below:

- Field Independence: the individuals tend to exhibit more individualistic behaviors since they are not in need of external referents to aide in the processing of information. They are more capable of developing their own internal referents and restructuring their knowledge, are better at learning impersonal abstract material, are not easily influenced by others, and are not overly affected by the approval or disapproval of superiors.

- Field Dependence: the individuals are considered to have a more social orientation than Field Independent persons since they are more likely to make use of externally developed social frameworks. They tend to seek out external referents for processing and structuring their information, are better at learning material with human content, are more readily influenced by the opinions of others, and are affected by the approval or disapproval of authority figures. (Witkin et al. 1977).

The Imagers/Verbalizer dimension characterizes the inclination of an individual to represent information during thinking either through mental pictures or verbally (Riding and Cheema, 1991). Their different characteristics are described below:

- Imagers: Individuals tend to be internal and passive. Imagers perform better if the environment presents text and also pictorial material such as pictures, diagrams, charts, and graphs. Imagers prefer to process information by seeing and they will learn most easily through visual and verbal presentations, rather than through an exclusively verbal medium.

- Verbalizers: Individuals tend to be external and stimulating. Verbalizers perform better if the environment presents only information in the form of text. Verbalizers prefer to process information through words and find they learn most easily by listening and talking (Laing, 2001).

These two dimensions of cognitive styles have been investigated by previous work in respect of the users' information seeking. In terms of Field Dependence/Independence, Palmquist and Kim (2000) investigated the effects of cognitive styles and online database search experience on Web search performance. The GEFT was administered to identify the cognitive styles of the 48 undergraduate college students. Their results showed that Field Dependent novices frequently made greater use of embedded links than other users. Furthermore, a more recent study by Ford et al. (2005) sought to discover the effects of human individual differences on Web search strategy. Their sample consisted of 250 master students and CSA was used to measure their cognitive styles. The results indicate that there is a link between low levels of Boolean searching (where individual search terms are combined using operators such as AND and OR) and Field Independence and a link between high levels of Boolean searching and Field Dependence.

In respect of Imager/Verbalizer, Graff (2005) examined differences in web browsing strategies between Imagers and Verbalizers. There were 58 participants, who read information in either a hierarchical or relational hypertext with the expectation of answering questions on this information. Differences between Imagers and Verbalizers are that the former visited more pages in the relational architecture whereas the latter visited more pages in the hierarchical architecture. In addition, Plass and Homer (2002) examined the preferences of Verbalizers and imagers in multimedia learning. 103 students using a German multimedia software were allowed to look up visual and verbal annotations for unknown words. The results showed that Imagers performed significantly better on words that reminded them of visual annotations than words that reminded them of verbal annotations, whereas Verbalizers 
showed the opposite pattern. Furthermore, Imagers performed better on propositions that allowed for visual and verbal annotations than on those that allowed only for verbal annotations, whereas Verbalizers performed well on both types of propositions.

The aforementioned studies provide interesting results. However, there is a paucity of studies, which investigate the preferences of these two dimensions of cognitive styles in DLs. In this paper, we attempt to provide a complete picture of the effects of cognitive styles on user preferences in DLs so both Field Dependence/Independence and Imager/Verbalize were investigated in this study, of which methodology design is described in next section.

\section{Methodology Design}

\subsection{Participants}

A total of 50 individuals participated in this study and such size of participants is in line with other studies in information seeking, e.g., Palmquist and Kim (2000). Participants were students at Brunel University in the United Kingdom and they volunteered to take part in the study. A request was issued to students in lectures, and further by email, making clear the nature of the studies and their participation. All participants had the basic computing and Internet skills necessary to operate the Brunel digital library catalog. In respects of Field Dependence/Independence, the sample consists of 18 Field Independent users, 21 Intermediate users, and 11 Field Dependent users. In respects of Imager/Verbalizer, there were 18 Imagers, 18 Bimodal, and 14 Verbalizers.

\subsection{Research Instruments}

Research instruments work as a guide in order to make sure that the same information is obtained from different participants. The research instruments used in this study included Riding's Cognitive Style Analysis, Brunel Library Catalogue, Webquilt for capturing user interaction, and two sets of questionnaires used for capturing user perception.

\subsubsection{Cognitive Styles Analysis}

The cognitive style dimensions investigated in this study include Field Dependence/Independence and Verbalizer/Imager. A number of instruments have been developed to measure these two dimensions. Riding's (1991) Cognitive Style Analysis (CSA) was applied to identify each participant's cognitive styles in this study because the CSA offers computerized administration and scoring.

The CSA uses two sub-tests to identify Field Dependence/Independence. The first presents items containing pairs of complex geometrical figures that the individual is required to judge as either the same or different. The second presents items each comprising a simple geometrical shape, such as a square or a triangle, and a complex geometrical figure, as in the GEFT, and the individual is asked to indicate whether or not the simple shape is contained in a complex one by pressing one of two marked response keys (Riding and Grimley, 1999).. The first sub-test is a task requiring Field Dependent capacity. Conversely, the second sub-test requires the disembedding capacity associated with Field Independence.

The CSA uses two types of statement to measure the Verbal-Imagery dimension and asks participants to judge whether the statements are true or false. The first type of statement contains information about conceptual categories while the second describes the appearance of items. It is assumed that Imagers respond more quickly to the appearance statements, because the objects can be readily represented as mental pictures and the information for the comparison can be obtained directly and rapidly from these images. In the case of the conceptual category items, it is assumed that Verbalizers have a shorter response time because the semantic conceptual category membership is verbally abstract in nature and cannot be represented in visual form. The computer records the response time to each statement and calculates the Verbal-Imager Ratio. A low ratio corresponds to a Verbalizer and a high ratio to an Imager, with the intermediate position being described as Bimodal. 
This study followed Riding's recommendation for the measurements of Field Dependence/Independence and Verbalizer/Imager. In terms of Field Dependence/Independence, Riding's (1991) recommendations are that scores below 1.03 denote Field Dependent individuals; scores of 1.36 and above denote Field Independent individuals; individuals scoring between 1.03 and 1.35 are classed as Intermediate. Regarding the measurement of Verbalizer/Imager, the recommendations are that scores below 0.98 denote Verbalizers; scores of 1.09 and above denote Imagers; individuals scoring between 0.98 and 1.09 are classed as Bimodal.

\subsubsection{Brunel Library Catalogue}

Brunel Library Catalogue (BLC) is a typical digital library and is used to access the bibliographical resources of Brunel University Library. The BLC has two main mechanisms that provide different strategies for finding information: (1) Basic Search (Figure 1a), which is the one presented by default by the system, and (2) Advanced Search (Figure 1b), which is accessed through the corresponding link presented in Figure 1a. Basic Search provides users with major search options, including "word or phrase", "author" "title" or "periodical title". Moreover, the users can choose in which library he/she wants to search for that information. The help link briefly describes what each link is supposed to do. Advanced Search, as presented in Figure 1b, presents the users with a much broader way of searching for information, including author, title, subject, periodical title, series, etc and combine these items using and/or Boolean operators. The Advanced Search also allows users to select other information, like the library, the language, the publication year, etc.

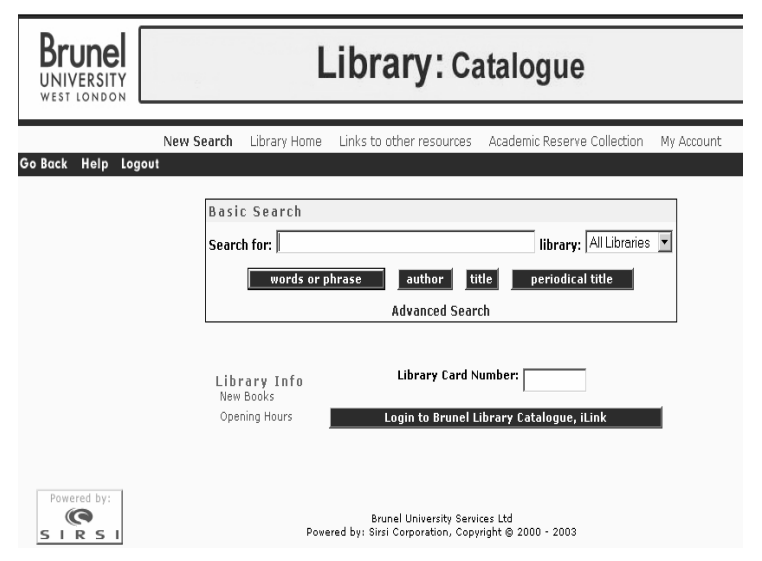

(a)

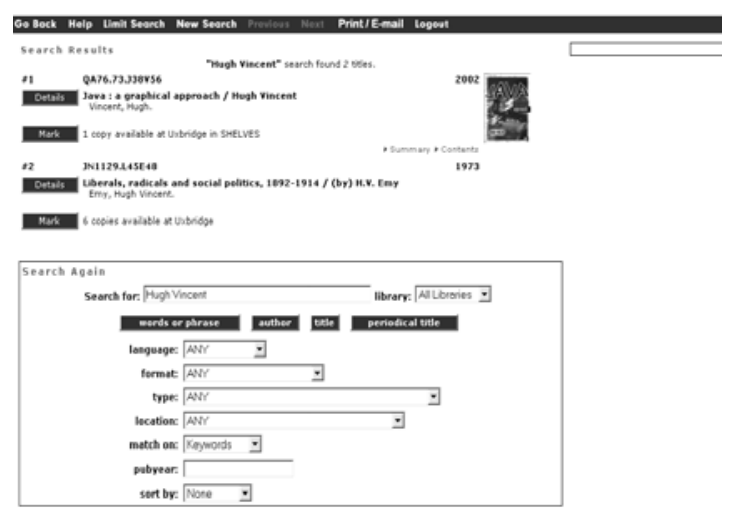

(c)

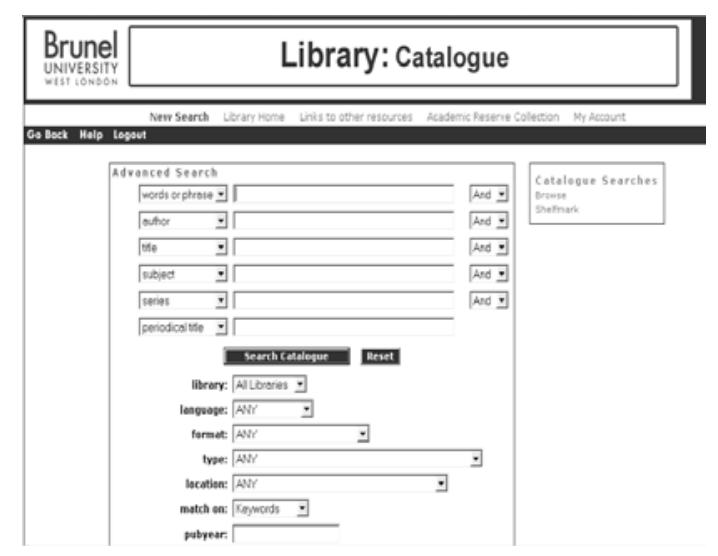

(b)

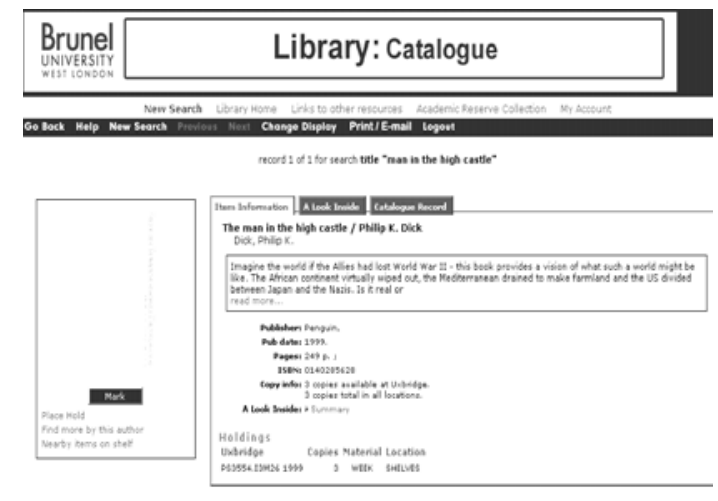

(d)

Fig. 1a). Basic Search Interface of BLC, (b) Advanced Search Interface of BLC, (c) Multiple Results Interface of BLC, and (d) Single Result Interface of BLC.

Once a user submits a query to the system using the Basic Search or the Advanced Search, the system responds with the items found in the database. An example of the interface presented is given in Figure 1c. The system presents a set of buttons in the top part: "Go Back", "Limit Search", "New Search", "Backward", "Forward", "Prefs" and 
"Exit". The "Limit Search" option is a link to the bottom of the page where the search mechanism used (Basic Search or Advanced Search) is presented with the terms used and a set of options for Search Limits (language, publication year, etc.). The limit search is obtained by adding more words to the set of terms already introduced. The "New Search" option presents again the interface of Figure 1a. The "Backward/Forward" button allows the user to move up and down the items found. Once a user selects one item, the information and interface given are presented as in Figure 1d.

\subsubsection{WebQuilt}

The WebQuilt Proxy Server (Hong et al. 2001) is a proxy system that unobtrusively gathers click stream data as users complete specified tasks. It is designed to conduct remote usability testing on a variety of Internet-enabled devices and provides a way to identify potential usability problems. WebQuilt utilizes Java Servlet and JSP technology to track users' interactions and then store that data by (1) creating a log file of each user's web use and (2) additionally caching the pages a user accesses for later viewing. Once the proxy server is running, each user connects to every web page through the web server. The proxy server stores any interaction between the user and any web pages and a snapshot of each page visited by each user. These snapshots are given a number that is the same one used to describe the sequence of pages visited by the user.

The Web proxy server has the possibility of adding a task box that can be used to indicate when a task has been finished. Once a user finishes each task and uses the task box links to finish it, Web Quilt provides each user with a set of questions regarding the task. All the information captured is stored in the proxy server and there is an identification number for each user. This allows centralizing all the information in the same place and at the same time being able to access the information of each user independently. The use of a proxy server architecture makes it easily capture all the interactions between users and the BLC.

\subsubsection{Questionnaires}

Two standardized questionnaires were used to capture user perception:

- Questionnaire for User Interface Satisfaction (QUIS) (Chin, Diehl \& Norman, 1988) has been designed to assess users' subjective satisfaction with specific aspects of the human-computer interaction. Although QUIS is a very complete questionnaire, for the purpose of this study a summarized QUIS comprising 27 questions was used. In this version, the questionnaire is divided into five sections: (1) Overall reaction to the software, (2) Screen, (3) Terminology/System Information, (4) Learning and (5) System Capabilities. Each area measures the users' overall satisfaction with that facet of the interface using a [0-9] scale.

- Computer Usability Questionnaire (CSUQ) (Lewis, 1995) was developed by IBM to evaluate the usability of a computer program (not necessarily a web service). It contains 19 questions, each one being a statement that the user has to rate on a [1-7] scale.

Table 1 and Table 2 present some examples of questions of CSUQ and QUIS respectively.

Table 1. Examples of CSUQ questions.

\begin{tabular}{cc}
\hline Question & Question \\
\hline 1 & I am satisfied with how easy it is to use the system: 1 (Strongly disagree) -7 (Strongly agree) \\
\hline 3 & I can effectively complete my work using this system: 1 (Strongly disagree) -7 (Strongly agree) \\
\hline 6 & I feel comfortable using this system: 1 (Strongly disagree) -7 (Strongly agree) \\
\hline 7 & It was easy to learn to use this system: 1 (Strongly disagree) -7 (Strongly agree) \\
\hline 16 & The interface of this system is pleasant: 1 (Strongly disagree) -7 (Strongly agree) \\
\hline 17 & I like using the interface of this system: 1 (Strongly disagree) -7 (Strongly agree) \\
\hline 18 & This system has all the functions I expect: 1 (Strongly disagree) -7 (Strongly agree) \\
\hline 19 & Overall, I am satisfied with this system: : 1 (Strongly disagree) -7 (Strongly agree) \\
\hline
\end{tabular}


Table 2. Examples of QUIS questions.

\begin{tabular}{ccc}
\hline Question & Question & Area \\
\hline 1 & The interface is: terrible (0) - wonderful (9) & Overall reaction to the software \\
\hline 2 & The interface is: Difficult (0) - Easy (9) & Overall reaction to the software \\
\hline 4 & The interface has: Inadequate Power (0) - Adequate Power (9) & Overall reaction to the software \\
\hline 6 & The system is: Rigid (0) - Flexible (9) & Overall reaction to the software \\
\hline 17 & Learning to operate the system is: Difficult (0) - Easy (9) & Learning \\
\hline 18 & Exploring new features by trial an error is: Difficult (0) - Easy (9) & Learning \\
\hline 27 & The system is for all level of users: Never (0) - Always (9) & System Capabilities \\
\hline
\end{tabular}

\subsection{Task Design}

This section describes the design of a set of tasks to capture the possible behavior that a user may have when interacting with the BLC. In the literature, usually two types of information seeking behavior are considered: (Bryan-Kinns, Blandford \& Thimbleby, 2000): browsing, which is defined as the finding of ill-defined information, and searching which is defined as the finding of well-defined information.

In order to characterize these types of behavior, volunteers were asked to perform a set of seven tasks. The set of tasks, presented in Table 3, was designed to involve all the different functionalities offered by the BLC. For example, question 1 (Table 3) captures a search behavior, because it has a well-defined answer contained in the library catalogue. This question is also designed to capture if the user uses the "Word or Phrase", "Author" or "Title" options or if an Advanced Search is used. The second task is a browsing question designed to test if the user chooses the "Subject" option or prefers an approach using "Title" or "Word or Phrase". The rest of the tasks are designed to replicate some of the functionalities and/or behavior, in order to have more relevant data to work with.

Table 3. Set of tasks designed for the experiment and their type.

\begin{tabular}{|c|c|c|}
\hline No. & Task & Type \\
\hline 1 & Find the Call Number of the book "The Man in the High Castle" by Philip Kendred Dick. & Search \\
\hline 2 & Find the title of any book related to applications of fuzzy logic. & Browse \\
\hline 3 & Find the number of books by Aldous Huxley that are part of the TWICKENHAM Library & Search \\
\hline 4 & Find a book about how to implement data mining with Java. & Browse \\
\hline 5 & Find a Java book written by Hugh Vincent. & Search \\
\hline 6 & Find a book about $20^{\text {th }}$ century American Drama in TWICKENHAM Library. & Browse \\
\hline 7 & Find an IEEE journal on consumer electronics. & Search \\
\hline
\end{tabular}

\subsection{Procedures}

In order to capture the behavior and perception of each user, the procedures below were followed in this study:

1) The CSA was used to identify participants' cognitive styles.

2) Participants were given a task sheet, containing the tasks presented in Table 3.

3) Participants conducted the tasks with the BLC. All interactions were stored by Webquilt.

4) Each participant answered QUIS and CSUQ questionnaires.

\subsection{Data Analyses}

In this study, the independent variable was the participants' cognitive styles, which include Field Dependence/Independence and Imager/Verbalizer. The dependent variable was user behavior and user perception.

- User Behavior. For each user, his/her behavior is summarized into seven dependant variables (variables 1 to 7 in Table 4) which have bee normalized "to one task" by dividing each value by seven. After normalization, Dimensions 1 to 7 capture the way in which each user interacts with the BLC to complete tasks. The behavior of each user was also characterized by the amount of time (Dimension 8 to 14) and the transactions needed to complete each task (Dimensions 15 to 21 in Table 4).

- User Perception: Dimensions 22 to 71 in Table 4 represent user perception and contain the 49 answers that each user gives to the QUIS and CSUQ questionnaires. 
Table 4: Dimensions of a BLC user vector.

\begin{tabular}{ccc}
\hline No. & Variable & Information \\
\hline 1 & BS & Number of times that Basic Search was used to solve a generic task. \\
\hline 2 & AS & Number of times that Advance Search was used to solve a generic task. \\
\hline 3 & SE & Number of times Word or Phrase was used to solve a generic task. \\
\hline 4 & ATS & Number of times that Author, Title and/or Periodical were used to solve a generic task. \\
\hline 5 & BF & Number of times that Backward/Forward was used to solve a generic task. \\
\hline 6 & NS & Number of times that New Search was used to solve a generic task. \\
\hline $8-14$ & GB & Number of times that Go Back was used to solve a generic task. \\
\hline $15-21$ & Trans(i) & Time in microseconds needed to complete task $i, i=1 \ldots 7$. \\
\hline $22-71$ & & Number of transactions needed to complete task $i, i=1 \ldots 7$. \\
\hline
\end{tabular}

\section{Results and Discussions}

\subsection{User Behavior}

The goal of this section is to analyze the behavior of DL users, with an attempt to answer the question, how cognitive styles affect user behavior in a DL. In this context, user behavior is defined as how users selected functionalities offered by the BLC, time required completing tasks, and transactions needed to complete tasks.

\subsubsection{Overall}

Table 5 presents, from a global perspective, the number of times that each functionality offered by the BLC has been used and the standard deviation. In general, a generic user interacts with the Basic Search (BS) option almost eight times more than with the Advanced Search (AS) option, while the Search Everything (SE) and the Author/Title/Periodical (ATS) buttons are used in the same proportion. This information can be also read literally as: A user that complete a task with the BLC, in average, uses the Basic Search (BS) 1.72 times and the Advance Search (AS) 0.31 times. Regarding the buttons, when using the Basic Search, Word or Phrase (SE) is used 0.63 times and the combination of Author, Title or Series (ATS) 0.77. The Backward/Forward (BF) button, the New Search (NS) button and the Go Back (GB) button are used 0.12, 0.15 and 0.17 times respectively. In summary, Basic Search $(B S)$ is the most popular functionality to users.

Table 5. Global Mean and Standard deviation of BLC user behavior.

\begin{tabular}{ccccccccc}
\hline & $\boldsymbol{B S}$ & $\boldsymbol{A S}$ & $\boldsymbol{S E}$ & $\boldsymbol{A T S}$ & $\boldsymbol{B F}$ & $\boldsymbol{N S}$ & 0.12 & 0.15 \\
\hline Mean & 1.72 & 0.31 & 0.63 & 0.77 & 0.17 & 0.03 & 0.06 & 0.03 \\
\hline Std. Deviation & 0.24 & 0.05 & 0.02 & 0.05 & & \\
\hline
\end{tabular}

Table 6. Global Mean and Standard deviation for the time and number of transactions needed.

\begin{tabular}{c|ccccc}
\hline & Time $(\mathrm{ms})$ & Trans & TimeSearch & TransSeacrh & TimeBrowse \\
\hline \multirow{2}{*}{ Mean } & 63656 & 5.12 & 55170 & 4.57 & 87452 \\
Std. Deviation & 14236 & 1.14 & 12682 & 0.8 & 22345 \\
\hline
\end{tabular}

Table 6 shows the time and number of transactions needed to complete the seven tasks. The firs two columns, Time and Trans, show the average time needed to conduct the seven tasks by all users and the average number of transactions respectively. The next two columns, TimeSearch and TransSearch, show the same information but focusing only on the search tasks (questions 1, 3, 5 and 7 of Table 3), while the last two columns TimeBrowse and TransBrowse show the information for the browsing tasks (questions 2, 4, and 6 of Table 3). Generally, a user takes 63 seconds to complete a task, but there is a big difference depending on the type of task. Search tasks are solved in only 55 seconds while browse tasks need to take 87 seconds. This considerable time difference between browsing and searching tasks is probably because the former was to find well-defined information while the latter was to find ill-defined information. More specifically, searching tasks contain the keywords needed to find information in the text that defines the task. For example, the searching task, "Find the Call Number of the book, The Man in the High Castle, by Philip Kendred Dick", already defines keywords for the title and the author. Nevertheless, browsing tasks are ill defined, so the user has to make more decisions about which keywords should be used. For example, the 
browsing task, "Find a book about $20^{\text {th }}$ century American Drama”, gives limited indications about how to select the keywords.

\subsection{Field Dependence/ Independence}

Tables 7 and 8 present the behavior based on Field Dependence/ Independence. The following summarizes some important behavioral differences between Field Dependent and Field Independent users:

Table 7. Behavior characteristics considering each FD/FI dimension (I).

\begin{tabular}{c|ccc}
\multicolumn{5}{c}{ Table 7. Behavior characteristics considering each FD/FI dimension (I). } \\
\hline & Field Dependent & Intermediate & Field Independent \\
\hline BS & 2.06 & 1.41 & 1.5 \\
AS & 0.01 & 0.29 & 0.84 \\
SE & 0.34 & 0.64 & 0.52 \\
ATS & 0.84 & 0.65 & 0.87 \\
BF & 0.25 & 0.01 & 0.01 \\
NS & 0.15 & 0.09 & 0.22 \\
GB & 0.2 & 0.2 & 0.08 \\
\hline \multicolumn{5}{c}{ Table 8. Behavior characteristics considering each FD/FI dimension (II). } \\
\hline AVTime & Field Dependent(ms) & Intermediate(ms) & Field Independent(ms) \\
AvTrans & 69732 & 49424 & 71813 \\
AvTimeSearch & 6.14 & 4.5 & 5.03 \\
AvTransSearch & 69932 & 42248.68 & 56392 \\
AvTimeBrowse & 5.37 & 4.54 & 4.68 \\
AvTransBrowse & 69582 & 54805.55 & 83380 \\
\hline
\end{tabular}

- $\quad$ Field Dependent users utilize only the Basic Search (BS) option combined with Author/Title/Periodical (ATS) and to some extent Word or Phrase (SE). Furthermore, it is notable the use of the Backward/Forward (BF) buttons.

- Intermediate users utilize mainly Basic Search (BS), although Advance Search (AS) plays also an important role in searching for information. Word or Phrase (SE) and Author/Title/Periodical (ATS) are used in the same proportion.

- $\quad$ Field Independent users utilize Basic Search (BS) as the main option and also rely more on Author/Title/Periodical (ATS) than on Word or Phrase (SE). There is a relevant use of the New Search (NS) button. Advance Search (AS) is also used although in a smaller proportion than Basic Search (BS).

The abovementioned results reinforce the literature regarding behavior differences between Field Dependent and Field Independent individuals (Chen and Macredie, 2002). In general, Field Dependent users prefer a linear approach to exploring the system, which justifies their use of the Backward/Forward button. Furthermore, Field Dependent users are more passive (Anastasi, 1988), which may explain the lack of use of the Advance Search option. On the other hand, Field Independent users tend to take an active approach (Goodenough 1976), which may also explain their use of the Advance Search option.

There are also considerable differences among each cognitive style when considering the time and transactions needed to complete a task. As showed in Table 8, Intermediate users complete a task faster than Field Dependent and Field Independent users and they also need fewer transactions to complete a task. Considering search and browse tasks separately, it is also true that Intermediate users spend less time and take fewer transactions in completing either searching or browsing tasks. One possible interpretation is that individuals possessing an Intermediate cognitive style combine the characteristics of both Field Independence and Field Dependence and employ a more versatile approach. Versatile users are more capable of adapting themselves to suit the design of a system (Ford, 2000) so they can effectively complete tasks with the BLC. Furthermore, it can be observed that there is not a big difference for Field Dependent users in the amount of time needed for a searching task and a browsing task. On the other hand, Field Independent users completed much faster for search tasks than browse tasks.

Furthermore, they also need fewer transactions for searching tasks than browsing tasks. This is probably because the descriptions of search tasks are provided with specific information, which are useful for Field Independent users who concentrated on procedural details when they process information (Chen and Macredie, 2004). 


\subsubsection{Verbalizer/Imager}

Table 9 and Table 10 present the behavior of Verbalizers and Imagers. As showed in Table 9, there are no important differences among these three cognitive styles. They use mainly Basic Search (BS), although Advance Search (AS) also plays an important role in all of them, especially for Verbalizers, who use it twice as much as Bimodals and Imagers. The rest of the functionalities are used in the same way by these three cognitive style groups.

\begin{tabular}{c|ccc}
\multicolumn{5}{c}{ Table 9. Behavior characteristics considering each V/I dimension (I). } & Imager \\
\hline & Verbalizer & Bimodal & 1.52 \\
BS & 1.37 & 1.75 & 0.2 \\
AS & 0.49 & 0.27 & 0.56 \\
SE & 0.44 & 0.77 & 0.74 \\
ATS & 0.64 & 0.85 & 0.02 \\
BF & 0 & 0.1 & 0.08 \\
NS & 0.2 & 0.19 & 0.16 \\
GB & 0.15 & 0.18 & \\
\multicolumn{5}{r}{} & & & Imager (ms) \\
\hline & Table 10. Behavior characteristics considering each V/I dimension (II). & 66742 \\
AvTime & Verbalizer(ms) & Bimodal(ms) & 4.52 \\
AvTrans & 56315 & 68154 & 57384 \\
AvTimeSearch & 5 & 5.4 & 4.2 \\
AvTransSearch & 45160 & 60137 & 73760 \\
AvTimeBrowse & 4.84 & 5.08 & 4.71 \\
AvTransBrowse & 64682 & 74167 & 5.73 \\
\hline
\end{tabular}

Although the interaction with the BLC is similar for these three types of cognitive style, there is an important difference in the time needed. Table 10 shows that Verbalizers spent considerably less time in completing a task than Bimodals and Imagers. This trend is also true when considering searching and browsing tasks separately. These results highlight the fact that the design of the BLC might match the preferences of Verbalizers. It may be due to the fact that the content is mainly text-based and there is a lack of multimedia elements. In other words, the inclusion of more multimedia elements in the BLC may help to improve the effectiveness of information seeking for the other two cognitive style groups.

\subsection{User Perception}

This section analyzes the perception of DL users, with an attempt to answer a question, how cognitive styles affect user perception in a DL. The results are presented for a selected subgroup of QUIS and CSUQ questions presented in Table 1 and Table 2 respectively. These questions are selected based on the semantic relevance to this study.

\subsubsection{Overall}

Table 11 and Table 12 present the global mean and standard deviation for the selected questions. It has to be noted that QUIS results are measured in a 0-9 scale while CSUQ questions are measured in a 1-7 scale. In general, the results from the QUIS questionnaires showed that users have a neutral opinion about the BLC (QUIS 1), that they think that the BLC is an easy tool to deal with (QUIS 2), that it is easy to learn to operate (QUIS 17), and that users also find the BLC a little bit rigid (QUIS 6). The results from the CSUQ questionnaires show that users have the perception that learning the system is simple (CSUQ 7) and that they feel comfortable using the BLC (CSUQ 6).

Table 11.Global Mean and Standard deviation of selected QUIS questions.

\begin{tabular}{c|ccccccc}
\hline & QUIS 1 & QUIS 2 & QUIS 4 & QUIS 6 & QUIS17 & QUIS 18 & QUIS 27 \\
\hline Mean & 5.23 & 6.63 & 5.13 & 4.87 & 6.43 & 5.67 & 5.27 \\
Std. Deviation & 1.300 & 0.903 & 1.417 & 1.300 & 1.161 & 1.591 & 1.518 \\
\hline
\end{tabular}


Table 12. Global Mean and Standard deviation for the selected CSUQ questions.

\begin{tabular}{|c|c|c|c|c|c|c|c|c|}
\hline & $\begin{array}{c}\text { CSUQ } \\
1 \\
\end{array}$ & $\begin{array}{c}\text { CSUQ } \\
3 \\
\end{array}$ & $\begin{array}{c}\text { CSUQ } \\
6 \\
\end{array}$ & $\begin{array}{c}\text { CSUQ } \\
7 \\
\end{array}$ & $\begin{array}{c}\text { CSUQ } \\
16 \\
\end{array}$ & $\begin{array}{c}\text { CSUQ } \\
17 \\
\end{array}$ & $\begin{array}{c}\text { CSUQ } \\
18 \\
\end{array}$ & $\begin{array}{c}\text { CSUQ } \\
19 \\
\end{array}$ \\
\hline Mean & 4.74 & 4.78 & 4.93 & 5.33 & 3.96 & 3.67 & 3.98 & 4.33 \\
\hline Std. Deviation & 1.443 & 1.525 & 1.439 & 1.274 & 1.636 & 1.554 & 1.548 & 1.467 \\
\hline
\end{tabular}

\subsubsection{Field Dependence/ Independence}

Table 13 and Table 14 present the perception based on Field Dependence/Independence. In general, Field Dependent users and Intermediate users are more satisfied with the BLC than Field Independent users. In particular, Field Dependent users and Intermediate users are happier with the power and flexibility (CSUQ 19) than Field Independent users. Regarding how simple is to use the BLC (QUIS 17), how difficult is to learn to use it (QUIS 18) and how comfortable a user feels using it (CSUQ 6), Field Dependent users find the BLC easier to operate and to learn than Field Independent and Intermediate users. It may be due to the fact that the simplicity of the BLC helps Field Dependent users to avoid the problem of feeling lost in hyperspace (Liu and Reed, 1994).

Table 13. Mean and Standard deviation for selected QUIS questions and FD/FI.

\begin{tabular}{|c|c|c|c|c|c|c|c|c|}
\hline \multicolumn{2}{|c|}{$F D / F I$} & $\begin{array}{c}\text { QUIS } \\
1\end{array}$ & $\begin{array}{c}\text { QUIS } \\
2\end{array}$ & $\begin{array}{c}\text { QUIS } \\
4\end{array}$ & $\begin{array}{c}\text { QUIS } \\
6\end{array}$ & $\begin{array}{c}\text { QUIS } \\
17\end{array}$ & $\begin{array}{c}\text { QUIS } \\
18\end{array}$ & $\begin{array}{c}\text { QUIS } \\
27\end{array}$ \\
\hline \multirow{2}{*}{ Field Independent } & Mean & 5.00 & 6.13 & 4.44 & 4.10 & 6.22 & 5.22 & 5.25 \\
\hline & Std. Deviation & 1.852 & 1.125 & 1.620 & 1.745 & 1.549 & 1.582 & 1.044 \\
\hline \multirow{2}{*}{ Intermediate } & Mean & 5.56 & 7.00 & 5.89 & 5.22 & 6.31 & 5.50 & 5.44 \\
\hline & Std. Deviation & 0.882 & 0.323 & 0.764 & 0.641 & 0.394 & 0.728 & 0.667 \\
\hline \multirow{2}{*}{ Field Dependent } & Mean & 5.40 & 6.90 & 4.90 & 4.75 & 7.20 & 7.00 & 5.00 \\
\hline & Std. Deviation & 1.408 & 1.236 & 1.881 & 1.074 & 1.168 & 1.449 & 1.345 \\
\hline
\end{tabular}

Table 14. Mean and Standard deviation for selected CSUQ questions and FD/FI.

\begin{tabular}{|c|c|c|c|c|c|c|c|c|c|}
\hline \multicolumn{2}{|c|}{$F D / F I$} & $\begin{array}{c}\text { CSUQ } \\
1\end{array}$ & $\begin{array}{c}\text { CSUQ } \\
3\end{array}$ & $\begin{array}{c}\text { CSUQ } \\
6\end{array}$ & $\begin{array}{c}\text { CSUQ } \\
7\end{array}$ & $\begin{array}{c}\text { CSUQ } \\
16\end{array}$ & $\begin{array}{c}\text { CSUQ } \\
17\end{array}$ & $\begin{array}{c}\text { CSUQ } \\
18\end{array}$ & $\begin{array}{c}\text { CSUQ } \\
19\end{array}$ \\
\hline \multirow{2}{*}{$\begin{array}{l}\text { Field } \\
\text { Independent }\end{array}$} & Mean & 4.76 & 4.67 & 4.76 & 4.95 & 3.95 & 3.57 & 3.52 & 4.00 \\
\hline & Std. Deviation & 1.080 & 1.083 & 1.011 & 1.003 & 1.096 & 1.099 & 1.004 & 1.012 \\
\hline \multirow{2}{*}{ Intermediate } & Mean & 4.71 & 4.75 & 4.96 & 5.62 & 3.96 & 4.54 & 4.38 & 4.54 \\
\hline & Std. Deviation & 0.488 & 0.567 & 0.488 & 0.209 & 0.546 & 0.641 & 0.583 & 0.318 \\
\hline \multirow{2}{*}{$\begin{array}{l}\text { Field } \\
\text { Dependent }\end{array}$} & Mean & 4.78 & 5.11 & 5.22 & 5.44 & 4.00 & 4.22 & 4.00 & 4.56 \\
\hline & Std. Deviation & 1.194 & 1.054 & 1.181 & 1.109 & 1.121 & 1.116 & 1.114 & 1.109 \\
\hline
\end{tabular}

In respect of functionality provided, Intermediate users have more positive perception than Field Dependent and Field Independent user. For example, they thought that the BLC has an adequate power (QUIS 4). Such positive perception may be the reason why they can take an effective way to locate information, especially spending less time and taking fewer transactions in completing tasks (Table 8). In other words, positive perception encourages them have better performance. These findings are in line with those of previous research (Chen, 2002), which highlighted that positive perception could enhance users' performance. In addition, the analyses of the standard deviation show that there is a more consistent opinion among Intermediate users than among Field Dependent and Field Independent users. The result reveals that most Intermediate users have similar perception to the design of the BLC.

Conversely, Field Independent users have more negative perception to the design of the BLC than Field Dependent and Intermediate users. In particular, they consider that the BLC is less flexible (QUIS 5) and does not provide that all the functions that they expect (CSUQ19). It is probably because Field Independent users are very task oriented (Witkin et al, 1977) so they expect that the BLC can provide more functionality for them to complete tasks. 


\subsubsection{Verbalizer/Imager}

Regarding the differences between Verbalizers and Imagers, (Table 15 and Table 16), the former are far more satisfied with the BLC than the latter (QUIS 1, 2, 4 and CSUQ 3, 6). In addition, Verbalizers consider the BLC more flexible than Imagers. It may be due to the fact that the BLC is mainly text-based and there is an absence of multimedia elements, which may be favored by Imagers. These results highlight those already found in Table 9 and Table 10, which showed that Verbalizers completed the tasks faster and with less number of transactions. Again, it provides another evidence to support the claim that positive perception to the design of systems can make users have better performance.

Table 15. Mean and Standard deviation for selected QUIS questions and Verbalizer/Imager.

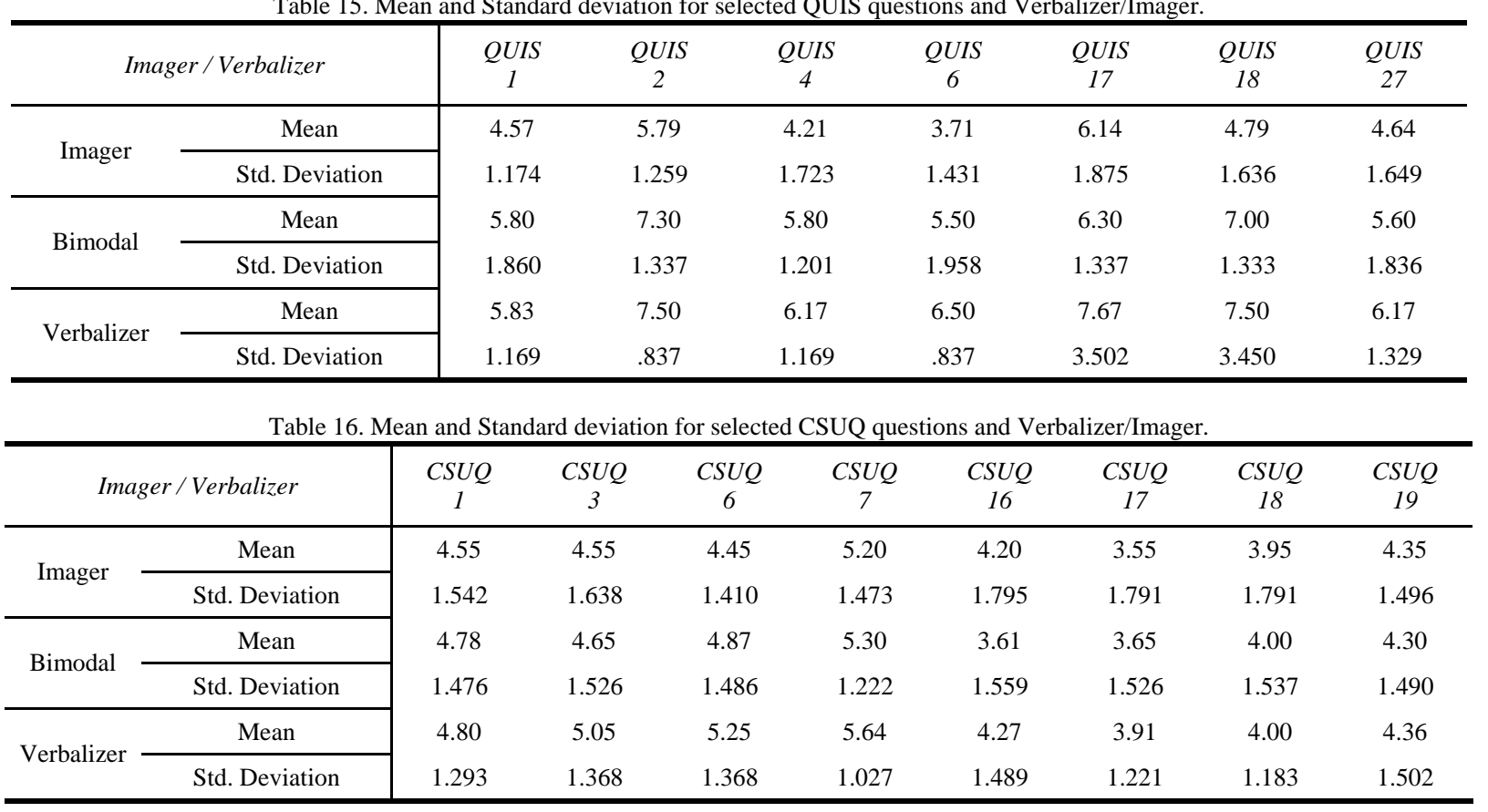

\section{Implications for System Design}

The results presented in Section 4 suggest that users' behavior and perception are greatly influenced by their cognitive styles. In general, Intermediate users and Verbalizers have more positive perception to the design of the BLC than other cognitive style groups. In addition, they conducted the tasks more effectively. However, the design of DLs cannot ignore the needs and preferences of other cognitive styles. For example, multimedia elements should be included in DLs so that the preferences of Imagers can be considered. Additionally, DLs should offer extra mechanisms for Field Independent users to complete tasks, for example, providing an alphabetical index to help them speed browsing tasks.

Nonetheless, such multimedia elements or extra mechanisms may not suit for other cognitive styles. Thus, there is a need to provide personalization. Personalization can be delivered by providing adaptivity or adaptability. With respect to the former, users' cognitive styles can be identified by either monitoring his/her behavior with data mining techniques or by obtaining this information from external surveys. According to their cognitive styles, the design of DLs could be automatically tailored to match with the preferences of each individual. With respect to the latter, users are allowed to modify the design of DLs by themselves. For example, a checkbox-based form can be applied to identify users' preferences. Each user will express his/her preferences by entering a checkmark in a suitable checkbox. The design of DLs will be changed based on their choices in the checkbox-based form. 


\section{Concluding Remarks}

This study examined the effects of cognitive styles on users' behavior and perception when they interacted with DLs. Two dimensions of cognitive styles were considered in this study: (a) Field Dependence/Independence; (2) Verbalizer/Imager. Riding's CSA was used to measure these two dimensions of cognitive styles. The results showed that Intermediate users and Verbalizers have not only more positive perception, but they also complete the tasks in effective ways. It suggests that positive perception can help users have better performance. Thus, future research should consider how to design DLs that can increase the satisfactions of different cognitive style groups, which, in turn, can positively influence their performance.

This study demonstrates the importance of cognitive styles in the use of DLs. However, it was only one such study. Further work needs to be undertaken with a larger sample to provide additional evidence. In addition, only two dimensions of cognitive styles are taken into account. Further works can have considerations for other dimensions of cognitive styles, e.g., Holism/Serialism. Furthermore, it would be interesting to run similar experiments in other DLs. The results of these studies can be integated with those of the study presented in this paper to build robust users models for the development of personalized DLs.

\section{References}

- Anastasi, A. (1988). Psychological testing. New York: Macmillan

- Ardissono, L., Goy, A., Petrone, G., and Segnan, M. (2005) A multi-agent infrastructure for developing personalized web-based systems. ACM Transactions on Internet Technology. 5(1), 47-69.

- Bryan-Kinns, N., Blandford, A., and Thimbleby, H. (2000). Interaction Modeling for Digital Libraries, Workshop on Evaluation of Information Management Systems.

- Chen, S. Y. (2002) The Relationships between Individual Differences and the Quality of Learning Outcomes in Web-based Instruction. Proceedings of the ICEB Second International Conference on Electronic Business, p. 345-351

- Chen, S. Y. and Macredie, R. D. (2002) Cognitive Styles and Hypermedia Navigation: Development of A Learning Model. Journal of the American Society for Information Science and Technology 53 (1) 3-15.

- Chen, S. Y. and Macredie, R. D. (2004) Cognitive Modelling of Student Learning in Web-based Instructional Programmes. International Journal of Human-Computer Interaction. 17(3), 375-402

- Chin, J.P., Diehl, V.A.,\& Norman, K.L. (1988). Development of an Instrument Measuring User Satisfaction of the Human-Computer Interface. ACM CHI'88 Proceedings, 213-218.

- Deshpande, M. and Karypis, G. (2004) Selective Markov models for predicting Web page accesses. ACM Transactions on Internet Technology. 4(2), 163-184

- Fink, J. and Kobsa, A. (2000) A Review and Analysis of Commercial User Modeling Servers for Personalization on the World Wide Web. User Modeling and User-Adapted Interaction, 9(34), 209-249

- Ford, N. (2000) Cognitive styles and virtual environments. Journal of the American Society for Information Science, 51(6), 543-557

- Ford, N., Miller, D. and Moss, N. (2005) Web search strategies and human individual differences: Cognitive and demographic factors, Internet attitudes, and approaches. Journal of the American Society for Information Science and Technology. 56, 741-756.

- Gonzalves, M.A, \& Fox, E.A. (2002). 5SL: a language for declarative specification and generation of digital libraries. In Proceedings of the 2nd ACM/IEEE-CS joint conference on Digital libraries Portland, Oregon, USA, $263-272$.

- Goodenough, D. (1976). The role of individual differences in field dependence as a factor in learning and memory. Psychological Bulletin, 83 , 675-694.

- Graff, M. (2005) Individual differences in hypertext browsing strategies. Behaviour and Information Technology, 24(2), 93-99

- Hong, J., Heer, J. Waterson, S., and Landay, J.A. (2001). WebQuilt: A Proxy-based Approach to Remote Web Usability Testing. ACM Transactions on Information Systems. 19, 263-385.

- Hudson, L. (1966) Contrary Imaginations, Penguin, Harmondsworth.

- Jonassen, D. H. and Grabowski, B. (1993) Individual Differences and Instruction. New York: Allen \& Bacon.

- Laing, M. (2001) Teaching Learning and Learning Teaching: An Introduction to Learning Styles. New Frontiers in Education 31(4), 463-475. 
- Lewis, J.R. (1995) IBM Computer Usability Satisfaction Questionnaires: Psychometric Evaluation and Instructions for User. International Journal of Human-Computer Interaction. 7, 57-78.

- Liaw, S. S. \& Huang, H. M. (2003) An investigation of users attitudes toward search engines as an information retrieval tool. Computers in Human Behavior. 19, 751-765.

- Liu, M., Reed, W.M. (1994) The effect of hypermedia assisted instruction on second-language learning through a semantic-network-based approach. Journal of Educational Computing Research. 12, 159-175.

- Marchionini, G., Plaisant, C. \& Komlodi, A. (1998). Interfaces and tools for the Library of Congress National Digital Library Program. Information Processing\&Management, 34(5), 535-555.

- Marchionini, G., Plaisant, C., \& Komlodi, A. (2003). The people in digital libraries: multifaceted approaches to assessing needs and impact. In Bishop, A., van House, N. A., Buttenfield, B.P. (Eds.) Digital Library Use Social Practice in Design and Evaluation. MIT Press, 119-160

- Messick S. (1976). Individuality in learning. San Francisco: Jossey-Bass.

- Mitchell, T. J. F., Chen, S. Y. and Macredie, R. D. (2005) Cognitive Styles and Adaptive Web-based Learning. Psychology of Education Review. 29(1), 34-42.

- Palmquist, R.A., \& Kim, K.S. (2000). Cognitive style and on-line database search experience as predictors of search performance. Journal of the American Society Information Science, 51(6), 558-566.

- Pask, G. (1976) Styles and strategies of learning. British Journal of Educational Psychology . 46, 128-148.

- Plass, J.L., \& Homer, B.D. (2002). Cognitive load in multimedia learning: The role of learner preferences and abilities. Paper accepted for presentation at the International Conference on Computers in Education (ICCE) in Auckland, New Zealand

- Riding, R. J. (1991). Cognitive Styles Analysis. Birmingham: Learning and Training Technology.

- Riding, R.J. \& Cheema, I. (1991). Cognitive Styles. Educational Psychology, 11(3/4), 193-215.

- Riding, R.J. and Grimley, M. (1999) Cognitive style and learning from multimedia materials in 11 year old children. British Journal of Educational Technology 30 (2) 43-56.

- Surjono, H. and Maltby, J. (2003). Adaptive educational hypermedia based on multiple student characteristics. Proceedings of the 2nd international conference on Web-based learning. Melbourne, Australia: LNCS-Springer Verlag.

- Witkin, H. A., Moore, C. A., Goodenough, D. R., \& Cox, P. W. (1977). Field-dependent and field independent cognitive styles and their educational implications. Review of Educational Research, 47( 1), 1-64. 